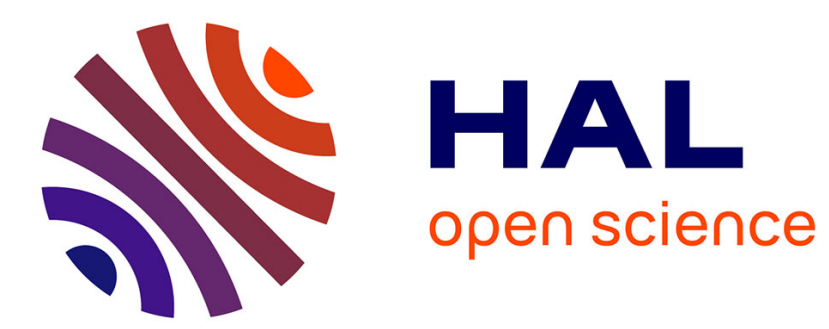

\title{
Excitation de la phosphorescence de CdI2 pur et dopé. Première Partie
}

Loic Langouet

\section{To cite this version:}

Loic Langouet. Excitation de la phosphorescence de CdI2 pur et dopé. Première Partie. Journal de Physique, 1967, 28 (7), pp.582-586. 10.1051/jphys:01967002807058200 . jpa-00206555

\section{HAL Id: jpa-00206555 https://hal.science/jpa-00206555}

Submitted on 1 Jan 1967

HAL is a multi-disciplinary open access archive for the deposit and dissemination of scientific research documents, whether they are published or not. The documents may come from teaching and research institutions in France or abroad, or from public or private research centers.
L'archive ouverte pluridisciplinaire HAL, est destinée au dépôt et à la diffusion de documents scientifiques de niveau recherche, publiés ou non, émanant des établissements d'enseignement et de recherche français ou étrangers, des laboratoires publics ou privés. 


\title{
EXGITATION DE LA PHOSPHORESGENGE DE GdI PUR ET DOPÉ Première Partie
}

\author{
Par LoḮ LANGOUET, \\ Laboratoire de Luminescence, Faculté des Sciences de Rennes.
}

\begin{abstract}
Résumé. - Par une étude de l'influence des traitements, du noircissement et de la température sur l'excitation de la phosphorescence de l'iodure de cadmium pur ou dopé, on a pu mettre en évidence une transition : bande de valence-bande de conduction, à la base du processus de phosphorescence de $\mathrm{CdI}_{2}$, définissant le caractère polymoléculaire de ce processus. Le dopage au mercure et à l'argent influence l'excitation de la phosphorescence.
\end{abstract}

Abstract. - A study of the influence of the preparation, of blackening and of temperature on the excitation of phosphorescence of $\mathrm{CdI}_{2}$ allowed us to distinguish a valence band conduction-band transition and to definite the centres of excitation. The character of the phosphorescence process is polymolecular. Doping by mercury and silver influence the excitation of phosphorescence.

I. Introduction. - Avec un montage expérimental, décrit par ailleurs [16], qui permet, grâce à un nouveau principe, d'enregistrer les spectres d'excitation de phosphorescence, nous avons pu étudier l'excitation de la phosphorescence de $\mathrm{CdI}_{2}$ pur et dopé, dans différentes conditions de préparation, de traitement et de température, et déterminer les transitions énergétiques des systèmes luminescents lors de cette excitation.

II. Enregistrement des spectres d'excitation de phosphorescence. - La grandeur caractéristique étudiée est l'intensité moyenne des courbes de déclin définie comme :

$$
l_{\mathrm{m}}=(1 / T) \int_{0}^{t} l(t) \mathrm{d} t
$$

$l(t)$ étant l'intensité de la phosphorescence en fonction du temps;

$T$ la durée de l'observation.

Voyons ce que représente $l_{\mathrm{m}}$. Puisqu'il est toujours possible de décomposer une loi de déclin $l(t)$ en une somme d'exponentielles décroissantes [17] :

$$
l(t)=\sum_{i=1}^{n} a_{i} \exp \left(-\lambda_{\mathrm{i}} t\right) .
$$

A partir de cette expression, on deduit $l_{\mathrm{m}}$ :

$$
l_{\mathrm{m}}=(1 / T) \sum_{i=1}^{n} a_{\mathrm{i}} / \lambda_{\mathrm{i}}-(1 / T) \sum_{i=1}^{n}\left(a_{i} / \lambda_{i}\right) \exp \left(-\lambda_{i} . T\right) \text {. }
$$

Mais au bout d'un long temps, il ne reste plus que l'exponentielle de plus petite constante $\lambda_{1}$ si bien que :

$$
\sum_{i=1}^{n}\left(a_{i} / \lambda_{i}\right) \exp \left(-\lambda_{i} T\right)
$$

se réduit à $\left(a_{1} / \lambda_{1}\right) \exp \left(-\lambda_{1} \cdot T\right)$.
En introduisant la somme de lumière :

$$
l_{m}=(1 / T) \sum_{i=1}^{n} S_{i}-(1 / T)\left(a_{1} / \lambda_{1}\right) \exp \left(-\lambda_{1} . T\right) .
$$

Lors de notre expérimentation, nous avons pris soin de prendre $T \gg 1 / \lambda_{1}$ :

$$
l_{\mathrm{m}} \simeq S / T \text {. }
$$

Mais on a la relation :

$$
S=A \cdot \eta\left(n_{\mathrm{p}_{0}}+n_{0}\right)
$$

$\eta$ étant le rendement de la transition;

$n_{\mathrm{p}_{0}}$ la population initiale du piège et $n_{0}$ celle de l'état excité émetteur;

$A$ un facteur de proportionnalité.

On peut admettre $n_{\mathrm{p}_{0}} \gg n_{0}$, d'où :

$$
l_{\mathrm{m}} \simeq(A / T) \eta \cdot n_{\mathrm{p}_{0}} \cdot
$$

L'intensité moyenne $l_{\mathrm{m}}$ est donc proportionnelle à la population des pièges à l'instant $t=0$. Le montage que nous avons mis au point pour mesurer $l_{\mathrm{m}}$ permet de faire des enregistrements de $l_{\mathrm{m}}$ pour chaque longueur d'onde excitatrice et d'obtenir ainsi les spectres d'excitation de phosphorescence.

III. Excitation de la phosphorescence de $\mathrm{CdI}_{2}$ pur. - III.1. ORIGINE DES PRODUITS ET PHOSPHORESGENCE. - L'iodure de cadmium peut être obtenu de plusieurs manières et parmi les principales, nous avons retenu [2] :

- La réaction de l'iodure de potassium sur le sulfate de cadmium, suivie d'un lavage à l'alcool.

- La réaction de l'iode sur le cadmium, soit à sec, soit en présence d'eau.

- La réaction de l'acide iodhydrique HI sur le cadmium. 
Nous n'avons pu savoir avec exactitude les procédés de fabrication des produits Rhône-Poullenc et Merck que nous avons utilisés. Toutefois, en faisant réagir de l'iodure de potassium sur du sulfate de cadmium, nous avons obtenu un produit dont l'analyse cristallographique a démontré une parfaite identité avec les produits du commerce.

Prolabo a bien voulu nous indiquer les caractéristiques moyennes d'analyse de l'iodure de cadmium pur RP fourni par ses soins :

\begin{tabular}{|c|c|}
\hline 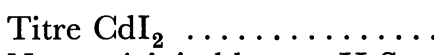 & 98 \\
\hline Non précipitable par $\mathrm{H}_{2} \mathrm{~S}$. & 0,5 \\
\hline Métaux lourds ........... & 0,010 \\
\hline $\mathrm{Cu} \ldots \ldots \ldots$ & 0,0020 \\
\hline & $0,0020 \%$ \\
\hline
\end{tabular}

Zn ............... Nul à l'essai.

Les spectres d'excitation de la phosphorescence de ces différents produits ont la même allure ( fig. 1); seules les intensités sont différentes et ces variations sont attribuables à des concentrations différentes des centres d'excitation.

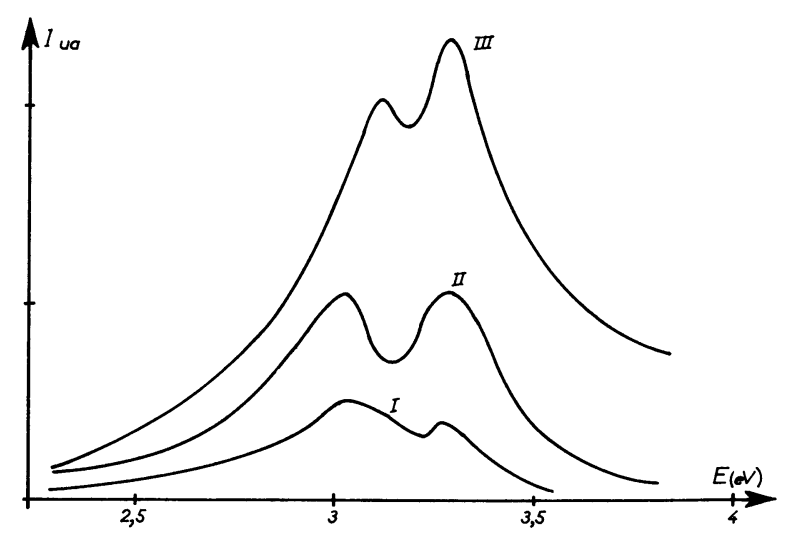

FIG. 1. - Excitation de la phosphorescence à $20^{\circ} \mathrm{C}$.

I : $\mathrm{CdI}_{2}$ pur Merck. - II : $\mathrm{CdI}_{2}$ pur Rhône-Poullenc "Prolabo ". - III : $\mathrm{CdI}_{2}$ pur par réaction de $\mathrm{KI}$ sur $\mathrm{CdSO}_{4}$.

Puisque les produits ont dû être préparés à partir de KI, nous avons étudié l'excitation de la phosphorescence de ce produit afin de voir si la phosphorescence de $\mathrm{CdI}_{2}$ ne pouvait pas être attribuée à des reliquats des produits de départ, mais $\mathrm{KI}$ ne présente, à $20^{\circ} \mathrm{C}$, aucune phosphorescence détectable. Ultérieurement, nous verrons que le dopage de $\mathrm{CdI}_{2}$ par le potassium n'influence pas l'excitation de la phosphorescence même à des températures plus basses; il en est de même pour le dopage par le cuivre, par le zinc et par les métaux lourds tels que le plomb et le thallium.

V. L. Levshin et S. G. Kroituru [3] ont observé que l'iodure de cadmium sublimé sous vide ne devenait luminescent qu'à l'ouverture de la chambre de préparation; ils ont attribué à l'oxygène l'appa- rition de la fluorescence jaune de ce produit, mais nous verrons que le phénomène de noircissement de $\mathrm{CdI}_{2}$ permet d'attribuer cet effet à la vapeur d'eau contenue dans l'air.

La phosphorescence semble donc étroitement liée à l'iodure de cadmium lui-même ou à ses constituants et non à des impuretés étrangères.

III.2. INFLUENGE DES TRAITEMENTS. - Nous avons voulu déterminer le rôle des traitements chimiques ou physiques que l'on est amené à faire subir à $\mathrm{CdI}_{2}$ pour préparer des produits dopés. Gette étude, réalisée à $20^{\circ} \mathrm{C}$, a porté sur le produit Prolabo en paillettes, sur un monocristal de $1 \mathrm{~mm}$ d'épaisseur ( ${ }^{\mathbf{1}}$ ) et sur six produits préparés respectivement par fusion, évaporation d'une solution aqueuse, broyage à sec, évaporation d'une solution dans l'acétone, évaporation d'une solution aqueuse en présence de cadmium et évaporation d'une solution dans l'alcool éthylique.

On note, pour chaque préparation, la présence d'un et parfois de deux maxima d'excitation, le premier aux environs de $3,02 \mathrm{eV}$ et le second vers $3,36 \mathrm{eV}$. Le produit en paillettes et le produit préparé par voie aqueuse ont le même spectre d'excitation de phosphorescence, tandis que, pour les autres préparations, on observe des différences d'intensité et de légères déformations des spectres. On peut proposer deux origines principales à ces variations :

- Une adsorption de certains solvants à la surface des cristaux d'iodure de cadmium [4].

- Des modes de cristallisation différents avec chaque préparation [2]; notamment le temps de cristallisation a une très grande influence sur la formation et la concentration des lacunes et des dislocations. Nous verrons, avec le phénomène de noircissement, l'influence particulière des faces des cristaux.

De plus, on note une similitude entre les courbes spectrales obtenues pour les produits fondus et pour ceux préparés par évaporation d'une solution aqueuse en présence de cadmium : les intensités sont particulièrement fortes dans les deux cas. Le dégagement de vapeur d'iode observé durant la fusion en tube non scellé nous faisait déjà supposer que la fusion constituait un dopage par le cadmium. En plus d'une confirmation de ce dopage, l'influence du dopage par le cadmium sur l'excitation de la phosphorescence est mise en évidence.

III.3. NoIRGissement ET EXGitATION DE PHOSPHORESGENCE. - Une longue irradiation de $\mathrm{CdI}_{2}$ par la lumière ultraviolette amène une modification des spectres d'excitation de phosphorescence de $\mathrm{CdI}_{2}$.

Pour le produit broyé à sec, lors de l'irradiation, on note un déplacement du maximum des courbes d'excitation et une augmentation de l'intensité des spectres. Le produit présente finalement un aspect

(1) Nous remercions vivement M. Le Donche qui a bien voulu préparer ce monocristal. 
blanc grisâtre que l'on peut facilement mettre en évidence en grattant la couche superficielle de la poudre.

Par contre, pour le produit en paillettes, cet effet de noircissement est très peu visible; cependant, l'excitation de la phosphorescence subit d'importantes modifications : l'intensité des spectres diminue et au lieu des deux maxima initiaux encadrant un minimum, après 20 heures d'irradiation, on n'observe plus qu'un seul maximum situé à l'emplacement du minimum précité.

La variation d'intensité des spectres est donc différente suivant que l'on a des paillettes ou des microcristaux. On peut expliquer ces effets par la création d'un écran absorbant à la fois passif et actif pour l'excitation de la phosphorescence, à la surface des cristaux. Avec les produits broyés, l'excitation se produit plus à la surface des préparations qu'avec les paillettes, à cause de la plus grande diffusion de la lumière excitatrice par les microcristaux. Les variations de l'absorption passive ont un rôle prépondérant pour les produits en paillettes (diminution des intensités) ; par contre, ce sont les variations de l'absorption active qui ont ce rôle pour les produits broyés (augmentation des intensités).

J. Hedvall, P. Walgren et S. Mausson [4] ont pu préciser que le noircissement se produisait sur les faces latérales des paillettes, lesquelles ont généralement une forme hexagonale liée aux modes de cristallisation. Le noircissement se produit en présence de vapeur d'eau et est attribuable à une dissociation totale ou partielle du produit sur ces faces latérales.

L'analyse des résultats publiés concernant la fluorescence de $\mathrm{CdI}_{2}(\mathrm{Cd})$ [5] et l'absorption des halogénures métalliques [6] semblent indiquer la présence de cadmium métallique en lui attribuant un rôle actif lors de la fluorescence et l'absorption de $\mathrm{CdI}_{2}$. De plus, compte tenu des structures cristallines de $\mathrm{CdI}_{2}$ et des dimensions de certaines sites particuliers, des inclusions de $\mathrm{Cd}$ ou de $\mathrm{Cd}^{+}$en position interstitielle sont très possibles à l'intérieur du cristal et a fortiori sur les faces des cristaux.

Notre étude expérimentale et bibliographique nous permet de penser que du cadmium $\mathrm{Cd}$ ou des ions $\mathrm{Cd}^{+}$, placés plus particulièrement sur les faces latérales des cristaux de $\mathrm{CdI}_{2}$, sont étroitement liés à l'excitation de la phosphorescence de $\mathrm{GdI}_{2}$.

Le noircissement n'existe pratiquement plus quand on dispose le produit sous vide; le cryostat [7] que nous avons utilisé permettait de maintenir le produit dans un vide de $10^{-3} \mathrm{~mm} \mathrm{Hg}$ et de ne pas être gêné par ce phénomène de noircissement.

III.4. VARiation AVEG LA température. - Nous avons enregistré, de $20^{\circ} \mathrm{G}$ à $-190^{\circ} \mathrm{G}$, les spectres d'excitation de phosphorescence de $\mathrm{CdI}_{2}$ pur broyé à sec. Au fur et à mesure que la température baisse, le spectre se déplace, en se déformant, vers les grandes énergies; on observe la présence de maxima et de minima, mais, alors que la position des maxima change considérablement, celle des deux minima observables surtout aux basses températures change relativement peu :

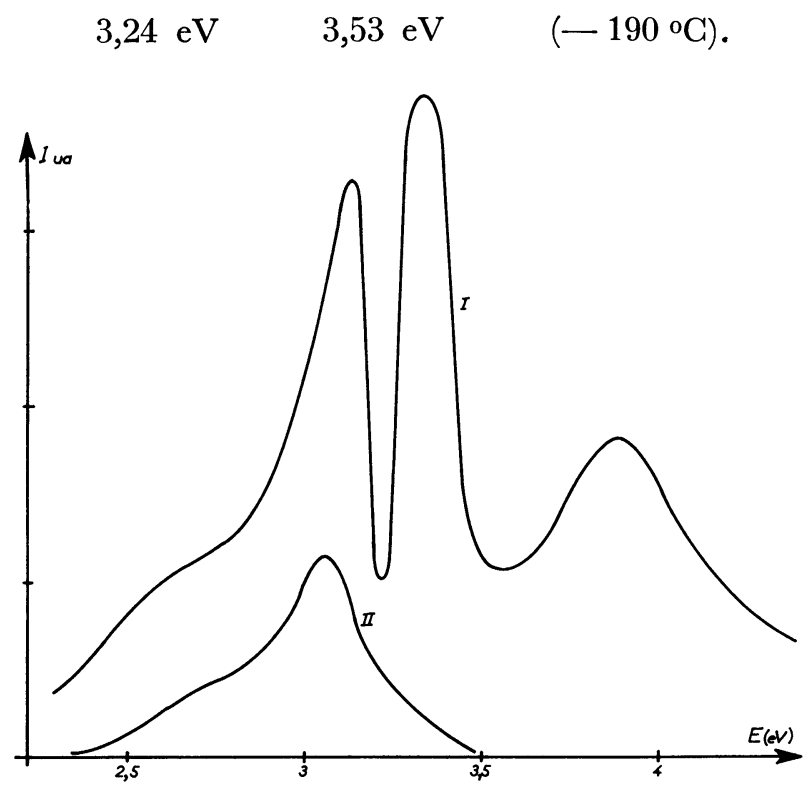

FIG. 2. - Excitation de la phosphorescence. $\mathrm{CdI}_{2}$ pur broyé à sec.

$$
\text { I : } T=-190^{\circ} \mathrm{C} \text {. }- \text { II }: T=20^{\circ} \mathrm{C} \text {. }
$$

La figure 2 donne les spectres d'excitation de phosphorescence de $\mathrm{CdI}_{2}$ pour $20^{\circ} \mathrm{C}$ et $-190^{\circ} \mathrm{C}$. Il nous a paru nécessairc de comparer ces spectres avec ceux de l'excitation de la fluorescence jaune de $\mathrm{CdI}_{2}$.

III.5. EXGitation DE LA FLUORESGENGE JAUNE DE $\mathrm{CdI}_{2}$. - Ces spectres ont pu être enregistrés de $-190^{\circ} \mathrm{G}$ à $-28^{\circ} \mathrm{G}$ avec le même produit broyé à sec; ces spectres se composent de deux bandes :

- la première de maximum : $3,24 \mathrm{eV}$ à $-190^{\circ} \mathrm{C}$ et $3,20 \mathrm{eV}$ à $-28^{\circ} \mathrm{C}$;

- la seconde de maximum : $3,53 \mathrm{eV}$ à $-190^{\circ} \mathrm{C}$ et $3,40 \mathrm{eV}$ à $-28^{\circ} \mathrm{C}$.

La largeur de ces bandes à mi-hauteur est faible : - pour la première bande : $0,06 \mathrm{eV}$ à $-190^{\circ} \mathrm{G}$ et $0,25 \mathrm{eV}$ à $-28^{\circ} \mathrm{C}$;

- pour la seconde bande : $0,14 \mathrm{eV}$ à $-190^{\circ} \mathrm{C}$ et $0,33 \mathrm{eV}$ à $-28^{\circ} \mathrm{C}$.

Quelle que soit la température, la position des maxima de ces deux bandes correspond :

- D'une part aux maxima des bandes des spectres d'absorption de $\mathrm{CdI}_{2}$ [8] attribuées à l'exciton $[9,10]$.

- D'autre part aux minima des spectres d'excitation de phosphorescence.

Les spectres d'excitation de fluorescence et de phosphorescence de $\mathrm{CdI}_{2}$ sont donc distincts. 
III.6. Exaitation DE LA PHOSPHORESCENGE DES halogénuRes mÉtalliques. - Parmi les halogénures métalliques proches de $\mathrm{CdI}_{2}$, nous avons cherché, sans succès, un rapport entre les spectres d'excitation de phosphorescence, le mode de cristallisation et les spectres d'absorption de tels produits.

III.7. Processus D'exaitation DE PHOSPhoresGENCE DE $\mathrm{CdI}_{2}$. - Les spectres d'excitation de phosphorescence et de fluorescence de $\mathrm{CdI}_{2}$ sont non seulement distincts mais concurrentiels, si l'on en juge par la correspondance entre les minima des premiers et les maxima des seconds. Il y a deux processus distincts qui coexistent lors de l'absorption de la lumière par $\mathrm{CdI}_{2}$. Le montage d'étude de la fluorescence étant le même que pour la phosphorescence (sauf pour la position du photomultiplicateur), nous avons pu déterminer le rapport entre les intensités de fluorescence et celles de phosphorescence : il est de 10000 environ à $-190^{\circ} \mathrm{C}$. En raison de cette valeur, lors de l'étude de la fluorescence, on ne détecte pas la phosphorescence qui est trop faible et, lors de l'étude de la phosphorescence, l'existence de la fluorescence se manifeste par une « modulation » du spectre d'excitation de phosphorescence.

Les spectres d'excitation de phosphorescence de $\mathrm{CdI}_{2}$ doivent être corrigés de cette absorption concurrente qui déforme ces spectres. Ils se présentent finalement sous la forme d'une bande unique de maximum :

$$
3,87 \mathrm{eV} \text { à }-190^{\circ} \mathrm{C} ; \quad 3,18 \mathrm{eV} \text { à } 20^{\circ} \mathrm{C} \text {. }
$$

D'autre part, les valeurs du GAP déterminées par différentes méthodes $[11,12]$ :

$$
3,07 \mathrm{eV} \text { à } 20^{\circ} \mathrm{C} ; \quad 3,47 \mathrm{eV} \text { à }-190^{\circ} \mathrm{C}
$$

correspondent à peu près au début de la bande d'excitation de phosphorescence du côté des faibles énergies. De plus, les énergies des spectres de photoconductivité mesurées à $20^{\circ} \mathrm{C}$ par Fotland [13] concordent avec celles de l'excitation de la phosphorescence de $\mathrm{CdI}_{2}$ : l'excitation de phosphorescence de $\mathrm{CdI}_{2}$ est donc due à une transition bande de valence-bande de conduction.

IV. Excitation de phosphorescence de l'iodure de cadmium dopé. - Nous avons tout d'abord préparé deux séries de produits :

- La première a été préparée par fusion; nous avons mélangé dans les proportions désirées l'iodure de cadmium et l'iodure du métal avec lequel nous voulions le doper. Alors que la température de fusion est de $387^{\circ} \mathrm{C}$, la température de préparation était imposée par la température de décomposition du moins fusible des deux iodures. On a ainsi obtenu 12 produits ayant une concentration en atomes de métal de dopage, par molécule de $\mathrm{CdI}_{2}$, de $10^{-2}$.

- La deuxième a été préparée par dissolution de l'iodure de cadmium et de l'iodure du métal dopant dans de l'eau distillée puis par évaporation de l'eau.
Tous les iodures n'étant pas solubles dans l'eau, nous n'avons pu préparer ainsi que 8 corps dopés dont la concentration en métal de dopage est de $10^{-3}$.

Seuls les produits dopés au mercure et à l'argent ont des spectres d'excitation de phosphorescence qui diffèrent de ceux obtenus avec $\mathrm{CdI}_{2}$ pur, si bien que ce sont les seuls dopages que nous avons étudiés en détail ultérieurement. Notons que le dopage par le plomb n'influe pas sur l'excitation de la phosphorescence, alors qu'il a une influence très importante sur l'excitation de la fluorescence jaune $[14,15]$.

IV.1. ExaitATion DE LA PHOSPHORESGENGE DE $\mathrm{CdI}_{2}(\mathrm{Hg})$. - Tous les produits dopés au mercure ont été préparés par voie aqueuse; quand la concentration de mercure augmente de $10^{-5}$ à $10^{-2}$, l'intensité des spectres de phosphorescence, à $20^{\circ} \mathrm{C}$, croît de manière homothétique : ce qui montre que le mercure joue un rôle dans les centres responsables de la phosphorescence.

A $20^{\circ} \mathrm{C}$, le spectre d'excitation de phosphorescence de $\mathrm{CdI}_{2}(\mathrm{Hg})$ comprend une bande unique perturbée par une absorption concurrente vers $3 \mathrm{eV}$ (fig. 3); quand la température s'abaisse, on note un déplace-

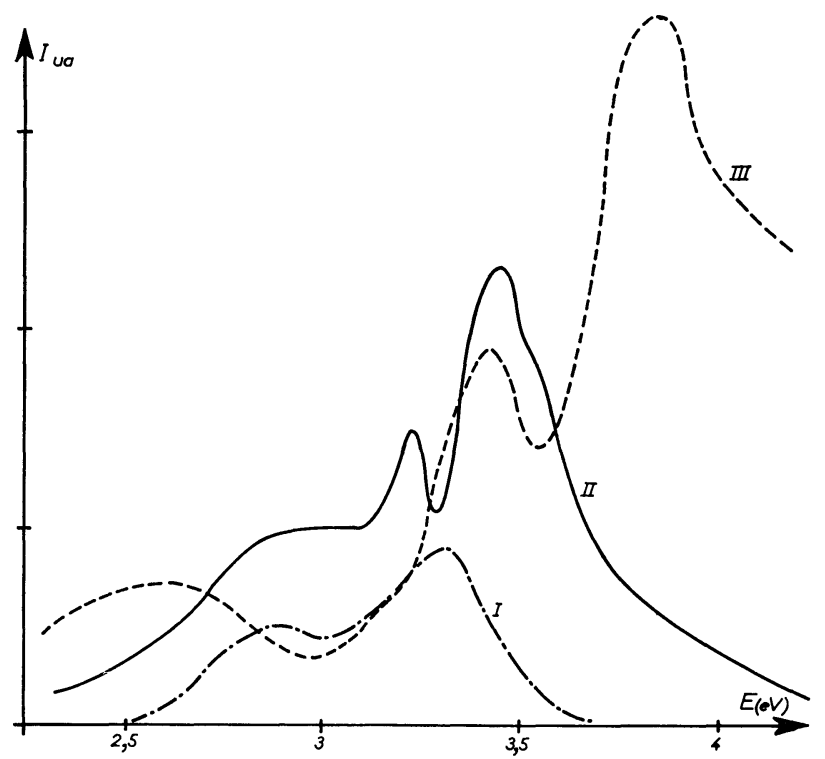

FIG. 3. - Excitation de la phosphorescence. $\mathrm{CdI}_{2}(\mathrm{Hg})$ préparé par évaporation d'une solution aqueuse : $c=10^{-3}$.

I : $T=20^{\circ} \mathrm{C}$. - II : $T=-100^{\circ} \mathrm{C}$. - III : $T=-190^{\circ} \mathrm{C}$.

ment du spectre du côté des grandes énergies et on n'observe l'apparition des mêmes minima que dans les spectres d'excitation de phosphorescence de $\mathrm{CdI}_{2}$ pur. En plus, entre $20^{\circ} \mathrm{C}$ et $-160^{\circ} \mathrm{C}$, une nouvelle bande de maximum 2,90 eV apparaît.

D'autre part, les spectres d'excitation de la fluorescence rouge de $\mathrm{CdI}_{2}(\mathrm{Hg})$ se composent de trois 
bandes : deux, déjà rencontrées avec $\mathrm{CdI}_{2}$ pur, et une nouvelle, qui est due à l'introduction du mercure dans $\mathrm{CdI}_{2}$. Cette dernière bande a son maximum vers $2,90 \mathrm{eV}$.

On peut noter que le spectre de phosphorescence de $\mathrm{Hg}_{2} \mathrm{I}_{2}$ comprend une bande unique de maximum $2,90 \mathrm{eV}$ et que celui de $\mathrm{HgI}_{2}$ (iodure utilisé pour le dopage) est différent avec une bande de maximum voisin de $2,2 \mathrm{eV}$.

Dans $\mathrm{CdI}_{2}(\mathrm{Hg})$, on a un nouveau processus d'excitation dû au mercure et peut-être plus précisément à des inclusions de $\mathrm{Hg}_{2} \mathrm{I}_{2}$.

IV.2. ExGITATION DE LA PHOSPHORESGENGE DE $\mathrm{CdI}_{2}(\mathrm{Ag})$. - L'iodure d'argent étant pratiquement insoluble dans l'eau, tous les produits dopés à l'argent ont été préparés par fusion à $550^{\circ} \mathrm{C}$. A la température ordinaire, le spectre de phosphorescence de $\mathrm{CdI}_{2}(\mathrm{Ag})$ est très proche de celui de $\mathrm{CdI}_{2}$, mais à basse température, du côté des faibles énergies, apparaissent de nouvelles bandes dont l'importance croît quand la concentration en argent augmente. Ces nouvelles bandes peuvent s'expliquer par une excitation, d'une part en surface, et d'autre part en profondeur, de AgI pur. Même à $-190^{\circ} \mathrm{C}, \mathrm{AgI}$ ne présente qu'une phosphorescence très faible; il semble donc falloir attribuer aux caractéristiques optiques d'absorption de $\mathrm{CdI}_{2}$ l'augmentation de l'intensité de cette phosphorescence dans $\mathrm{CdI}_{2}(\mathrm{Ag})$.
Ainsi, il semble que, dans $\mathrm{CdI}_{2}(\mathrm{Ag})$, on ait une juxtaposition d'iodure de cadmium et d'iodure d'argent, et que ce mélange favorise l'excitation de la phosphorescence de AgI.

V. Conclusion. - Cette étude de l'excitation de la phosphorescence de $\mathrm{CdI}_{2}$ a permis de localiser sur les faces latérales des paillettes hexagonales les centres d'excitation et d'attribuer ces centres à du cadmium Cd ou à des ions $\mathrm{Cd}^{+}$. Lors de ce processus d'excitation, un électron passerait de la bande de valence dans la bande de conduction; en admettant qu'il vienne se fixer dans des centres $\mathrm{Cd}^{+}$qui deviennent ainsi des centres $\mathrm{Cd}$, on explique la présence de $\mathrm{Cd}^{+}$et de $\mathrm{Gd}$ et leur rôle dans les excitations de fluorescence et phosphorescence et dans l'émission de fluorescence de $\mathrm{CdI}_{2}$.

Les effets du dopage au mercure, ou à l'argent, peuvent s'expliquer par une juxtaposition de $\mathrm{CdI}_{2}$ et de $\mathrm{Hg}_{2} \mathrm{I}_{2}$ d'une part, et de AgI d'autre part. Alors que ces derniers iodures ont une phosphorescence de faible intensité à l'état pur, lorsqu'ils sont insérés dans le réseau de $\mathrm{CdI}_{2}$, cette phosphorescence augmente; les centres d'excitation, qui existent déjà dans les iodures dopants à l'état pur, sont plus excitables dans l'iodure de cadmium dopé.

Manuscrit reçu le 25 novembre 1966.

\section{BIBLIOGRAPHIE}

[1] Résumé d'une partie d'une thèse de doctorat d'État (Sciences-Physiques), Rennes, 1967.

[2] PASCAL, Zinc, Cadmium et Mercure, tome V, 356-361.

[3] LEVShin (V. L.) et Kroituru (S. G.), opt. y. Spectr., 1964, 2, 492.

[4] Hedval, (J.), Walgren (P.) et Mausson (S.), Trans. Far. Soc., 1940, 36, 697-706.

[5] Chalimova (K. V.) et Bielikova (T. P.), Dokl. Akad. Nauk. SSSR, 1952, 82, 5.

[6] FESEFELDT (H.), Z. Physik, 1930, 64, 741.

[7] Monod-Herzen (G.), T'KINT DE RoOdenbeke (A.) et Giquelais (H.), J. Physique Rad., 1962, 23, 147 A.
[8] Gross (E. F.) et Kapliansky (A. A.), J. Tech. Phys. SSSR, 1955, 25, 2061.

[9] Dresselhaus (G.), Phys. Rev., 1957, 106, 1, 76-78.

[10] Gross (E. F.), J. Physique Rad., 1956, 17, 815-816.

[11] Chapiro (I. P.), Dokl. Akad. Nauk. SSSR, 1961.

[12] NIILISK (A. I.), HERST (A. V.) et KIRS (J. J.), Fiz. Tverd. Tela, SSSR, 1965, 3, 7, 931-933.

[13] Fotrand (R. A.), J. Chem. Physics, 1960, 33, 956.

[14] Monod-Herzen (G.) et Le Calvez (O.), C. R. Acad. Sc., 1962, 254, 4160.

[15] Monod-Herzen (G.), Demay (M. F.) et LaNGOUET (L.), C. R. Acad. Sc., 1965, 260, 3343-3344.

[16] Langoue'T (L.), Bull. Soc. Sc. Bret., 1966, 41, 314.

[17] SADDy (J.), J. Physique Rad., 1959, 20, 890-897. 